\section{IDDF2020-ABS-0075 OPTIMIZING THE USE OF GASTROSCOPE FOR ICU PATIENTS BASED ON MACHINE LEARNING MODEL}

Yi Yu*. The Second Affiliated Hospital of Guangzhou University of Chinese Medicine, China

\subsection{6/gutjnl-2020-IDDF.62}

Background We aim to establish an objective and feasible pregastroscopic screening standard to solve the overuse of gastroscopy for ICU patients.

Methods This study collected the demographic information, diet, lifestyle, medical history, symptoms, PGI, PGII, G-17 and $\mathrm{Hp}$ antibody of the patients from the MIMIC-III and Philips eICU collaboration databases. The decision tree model, logistic regression model, random forest model and support vector machine model were trained by the collected information. The accuracy and validity of the machine learning models predicting positive gastroscopic results were evaluated by comparing the efficiencies of different pre-gastroscopic screening ways.

Results 1273 gastroscopic positive cases of a total of 720 cases were enrolled in this study. In the training set, support vector machine model fitted the highest degree (AUC=1.000), the random forest model $(\mathrm{AUC}=0.941)$, the decision tree model (AUC is 0.885 ), and the worst is the Logistic regression model (AUC $=0.839)$. In the test set, four machine learning model has better prediction effect, AUC from high to low were random forest model (0.879), logistic regression model (0.842), the decision tree model $(0.827)$ and support vector machine model $(0.826)$. Assuming risk cut-off value was 0.85 , the sensitivity of the model is $93.17 \%$, as well as specificity is $15.70 \%$, and only recommended gastroscopy in $89 \%$ of patients, the average 2.27 times gastroscopy can be found that the positive cases. Compared with direct gastroscopy, the efficiency of gastroscopy is increased by 3.57 times after using the screening model.

Conclusions This study compared the variables in the model with single-factor analysis results, and proved that the history of upper gastrointestinal polyps, PG II, PG I, Hp antibody, smoking, drinking were important predicting variables for positive gastroscopic results, as well as the single alarm symptom is difficult to predict the results of gastroscope accurately. The model can predict positive gastroscopic risk effectively and provide objective criteria for optimizing the use of gastroscope, which may be a new way to decrease the overuse of gastroscopy for ICU patients. However, before being applied in clinical practice, the models need externally validated.

\section{IDDF2020-ABS-0076 DIAGNOSTIC VALUE OF DOUBLE-BALLOON ENTEROSCOPY, CT ENTEROGRAPHY AND THEIR COMBINATION FOR SMALL BOWEL CROHN DISEASE}

${ }^{1}$ Xiaodong Chen*, 'Jiachuan Wu, ${ }^{2}$ Zhijian Li, ${ }^{1}$ Ruiyan Chen, 'Xiaoqiao Yang, ${ }^{1}$ Biao Liang, ${ }^{1}$ Lifang Ye, 'Lili Ye, ${ }^{1}$ Haizhu Li. ' Guangdong Second Provincial General Hospital, China; ${ }^{2}$ Shunde Hospital of Southern Medical University, China

\subsection{6/gutjnl-2020-IDDF.63}

Background To investigate the value of double-balloon endoscopy(DBE), CT enterography (CTE) and their combination for the diagnosis of small bowel Crohn disease(CD).

Methods Data of 35 patients admitted from January 2018 to December 2019 who were clinically suspected of small bowel CD undergoing double-balloon endoscopy(DBE) were reviewed. 30 patients underwent CT enterography (CTE). Diagnosis was made based on pathological, endoscopic findings, CT enterography findings and clinic follow-up results. Detection rates and diagnosis rates of small bowel CD were compared by double-balloon enteroscopy(DBE) and CT enterography(CTE).

Results The detection and diagnosis rates of small bowel CD by double-balloon endoscopy (DBE) were 97.1\%(34/35) and 91.2\%(31/34), respectively. These two variables by CT enterography(CTE) were $86.7 \%(26 / 30)$ and $88.5 \%(23 / 26)$. Both double balloon enteroscopy (DBE) and CT enterography (CTE) detected small intestinal CD in 25 cases, with a detection rate of $83.3 \%(25 / 30)$ and a diagnosis rate of $92 \%(23 /$ $25)$. The detection rate and diagnosis rate of double balloon enteroscopy (DBE) were higher than that of CT enterography (CTE). Double balloon enteroscopy(DBE) combined with CT enterography(CTE) has the highest rate of diagnosis.

Conclusions Double balloon enteroscopy(DBE) has high application value for the diagnosis of small bowel CD, and can be recommended as the first choice for the diagnosis of small bowel CD. For those contraindicated with endoscopy, CT enterography(CTE) can be considered as a preferred auxiliary diagnostic modality. The combination of the two methods can complement each other and provide more information for the diagnosis of small bowel CD, thus improving the diagnosis rate of small intestinal CD.

\section{IDDF2020-ABS-0077 TREATMENT PATTERNS SHOULD BE CAREFULLY CHOSEN IN DIFFERENT PRIMARY SITES OF GI-NECS}

${ }^{1}$ Wen Cai* ${ }^{*}$ ' Weiting Ge, ${ }^{2}$ Dehao Wu, ${ }^{1}$ Jianshan Mao, ${ }^{3}$ Hanguang Hu. ${ }^{1}$ Department of Gastroenterology, Second Affiliated Hospital of Zhejiang University School of Medicine, China; ${ }^{2}$ Cancer Institute (Key Laboratory of Cancer Prevention and Intervention, China National Ministry of Education), the Second Affiliated Hospital, School of Medicine, Zhejiang University, China; ${ }^{3}$ Department of Medical Oncology, the Second Affiliated Hospital, School of Medicine, Zhejiang University, China

\subsection{6/gutjnl-2020-IDDF.64}

Background Neuroendocrine carcinomas (NECs) are heterogeneous and aggressive in gastrointestinal tract (GI). However, treatment patterns and related outcomes in the different primary site have not been well described.

Methods The SEER data was selected from 2010 to 2016, and 5 -year survival was set as the end-point. Coarsened exact matching (CEM) was performed to adjust before further regression models. Patients were separated by treatment groups and then comparing survivals for treatment patterns used multivariate analysis in different primary sites. Patients with nonchemotherapy and non-surgery were considered as the reference group.

Results 4114 patients with GI-NECs including stomach (12.96\%), small intestinal (37.50\%), colon (24.45\%) and rectum $(25.09 \%)$ were identified. In the stomach, chemotherapy without surgery will increase the risk of death in non-metastatic NEC patients $(\mathrm{HR}=3.11,95 \%$ CI $1.26-7.76 ; P=0.014)$. Chemotherapy combining with primary resection will benefit metastatic patients $(\mathrm{HR}=0.15,95 \% \mathrm{CI} 1.26-7.76 ; P=0.017)$. In small intestinal, single primary resection will benefit both non-metastatic $(\mathrm{HR}=0.67,95 \% \mathrm{CI} \quad 0.45-0.98 ; P=0.042)$ and metastatic $(\mathrm{HR}=0.61,95 \% \mathrm{CI} \quad 0.41-0.92 ; P=0.018)$ patients younger than 60 -year-old. In the colon, primary site resection combines with chemotherapy will benefit the metastatic patient 
$(\mathrm{HR}=0.41, \quad 95 \% \mathrm{CI} \quad 0.18-0.95 ; \quad P=0.039) \quad$ comparing with other therapy combination. In the rectum, combining chemotherapy, radiotherapy, and primary surgery in non-metastatic patients will increase the risk of death $(\mathrm{HR}=2.11$, 95\% CI 1.14-4.00; $P=0.022$ ). Pooling all patients received metastatic sites resection and comparing with the reference group, metastatic sites resection in GI-NEC will bring survival benefits ( $\mathrm{HR}=0.42$, 95\%CI 0.19-0.93; $P=0.033)$.

Conclusions GI-NECs have different treatment patterns. Primary sites resection should be the basic treatment choices for GI-NECs. Chemotherapy should be cautious, especially in non-metastatic patients and considered more biological characteristics of NECs (eg: Ki-67) before using it. Patients with distant metastasis can benefit from metastatic sites resection.

\section{IDDF2020-ABS-0079 A SINGLE CENTRE RETROSPECTIVE STUDY OF INPATIENT MANAGEMENT IN ACUTE LOWER GASTROINTESTINAL BLEEDING}

Wilson Siu*, Yusuke Onishi, Wamedh Taj-Aldeen, Balasubramaniam Vijayan. Department of Digestive Disorders, Aberdeen Royal Infirmary, UK

\subsection{6/gutjnl-2020-IDDF.65}

Background Acute lower gastrointestinal bleeding (ALGIB) is a common presenting condition in hospital with an estimated incidence of $33-87 / 100000$. Recent national audit in the United Kingdom has shown that the bleeding stops in the majority of the cases without any intervention. In this retrospective study, we aim to describe patient characteristics and to identify factors that predict clinical outcomes.

Methods Haemodynamically unstable patients with ALGIB are admitted to the medical high dependency unit (MHDU) at Aberdeen Royal Infirmary for monitoring. Patients with a primary diagnosis of ALGIB between 01/05/2015 to 15/09/2017 were identified from the MHDU database. Patients who presented with haematemesis or had upper gastrointestinal (UGI) bleeding found at esophagogastroduodenoscopy were excluded. Patient's demographic data, laboratory results, medications, endoscopy and radiology reports were collected. Clinically relevant outcomes of the study included 28-day mortality and red cell transfusion requirement. Multivariable logistic regression analysis was used to identify factors independently associated with outcomes.

Results 130 patients (Median Age 73; male predominance $68 \%$ ) were included in the study after excluding readmissions $(\mathrm{n}=8)$ and UGI bleedings $(\mathrm{n}=9) .51 \%$ had major comorbidity, $37 \%$ taking antiplatelets and 25\% taking anticoagulants. $60 \%$ received blood transfusion and $31 \%$ required intervention (endoscopic therapy $(n=17)$, mesenteric embolization $(n=18)$ and surgery $(n=5))$. $72 \%$ had diagnostic endoscopy on admission with the majority being flexible sigmoidoscopy $(n=74)$. Median Length of hospital stay was 6 days, and 12\% experienced rebleeding on the same admission. 10 patients died within 28 days of admission. Low Haemoglobin $(p=0.027)$, raised C-reactive protein $(\mathrm{CRP})(\mathrm{p}=0.047)$ and no endoscopy performed on admission $(p=0.014)$ were associated with 28 day mortality. Low Haemoglobin $(\mathrm{p}<0.0001)$ was also significantly associated with red cell transfusion requirement.

Conclusions In our study, the majority of patients who were admitted with severe ALGIB were elderly with a high burden of co-morbidities and frequent antithrombotic use. Nevertheless, antithrombotic medication and co-morbidities were not significantly associated with mortality or red cell transfusion requirement.

\section{IDDF2020-ABS-0082 LIGHT WEIGHT PROLENE MESH WAS ASSOCIATED WITH LOWER INCIDENCE OF MESH REJECTION ON INGUINAL HERNIA UNDERGOING OPEN EMERGENCY HERNIA SURGERY}

${ }^{1}$ Budhi Ida Bagus*, ${ }^{2}$ Metria Ida Bagus, ${ }^{3}$ Setyawati Ida Ayu, ${ }^{1}$ Soewoto Widyanti, ${ }^{1}$ Wibisono Wibisono, ${ }^{1}$ Setyono Hanis, ${ }^{1}$ Ismail Darmawan, ${ }^{1}$ Yuli Yarso Kristanto. ${ }^{1}$ Department of Surgery, Sebelas Maret University, Indonesia; ${ }^{2}$ Medical Faculty, Sebelas Maret University, Indonesia; ${ }^{3}$ Medical Faculty, Pendidikan Ganesha University, Indonesia

\subsection{6/gutjnl-2020-IDDF.66}

Background Incarserated hernia was one of the most common procedures in gastrointestinal surgery. According to the latest Hernia Guidelines 2018, emergency hernia surgery has been suggested to used mesh in cases without contamination, whether we did by laparoscopic or open conventional procedures, but there was lack of data and recommendation, which kind of mesh should we used which could reduce the incidence of mesh rejection during emergency hernia surgery.

Methods Incarserated hernia cases which have been done open conventional hernia repair procedure (Lichtenstein procedure) were included in this study during Januari-December 2019. The patients were divided into two groups; the first group would use light-weight prolene mesh and the other one using heavy-weight mesh. The outcome that would be evaluated was the incidence of mesh rejection during those procedures after 6 months follow up. The cases with the presence of contamination would be excluded from this study.

Results 45 patients were included in this study, 24 patients with light-weight prolene mesh following Lichtenstein procedure and 21 patients using the heavy-weight mesh. During the follow-up, there was no drop out of the patient, and during 5 months follow up, we found 1 case of mesh rejection, 2 months after those procedures using heavy-weight mesh and had been managed by the operative procedure for debridement of the mesh rejection. On the other hand, we found no mesh rejection on light-weight mesh group $(\mathrm{p}<0.05)$.

Conclusions Light-weight prolene mesh was associated with a lower incidence of mesh rejection during open emergency hernia surgery.

\section{IDDF2020-ABS-0083 THE LENGTH OF STAY DIFFERENCE BETWEEN OPEN CONVENTIONAL VS LAPAROSCOPY CHOLECYSTECTOMY ON CALCULOUS CHOLECYSTITIS PATIENTS IN THE CURRENT GOVERNMENT INSURANCE ERA}

${ }^{1}$ Budhi Ida Bagus*, ${ }^{2}$ Metria Ida Bagus, ${ }^{3}$ Setyawati Ida Ayu, ${ }^{4}$ Mastini Ida Ayu Kade. ${ }^{1}$ Department of Surgery, Sebelas Maret University, Indonesia; ' 2 Medical Faculty, Sebelas Maret University, Indonesia; ${ }^{3}$ Medical Faculty, Pendidikan Ganesha University, Indonesia; ${ }^{4}$ Clincal Pharmacy Department, Moewardi General Hospital, Indonesia

\subsection{6/gutjin-2020-IDDF.67}

Background For the last two decades, laparoscopy cholecystectomy has been announced as the gold standard procedure for symptomatic cholelithiasis, and calculous cholecystitis with the main benefits was less post-operative pain and shorter length 